\title{
Coupling between Transverse Vibrations and Instability Phenomena of Plates Subjected to In-Plane Loading
}

\author{
D. V. Bambill ${ }^{1,2}$ and C. A. Rossit ${ }^{1,2}$ \\ ${ }^{1}$ Department of Engineering, Institute of Applied Mechanics (IMA), Universidad Nacional del Sur (UNS), \\ Alem 1253, B8000CPB Bahía Blanca, Argentina \\ ${ }^{2}$ Consejo Nacional de Investigaciones Científicas y Técnicas, (CONICET), Bahía Blanca, Argentina
}

Correspondence should be addressed to D. V. Bambill; dbambill@criba.edu.ar

Received 27 November 2012; Accepted 31 January 2013

Academic Editor: Gabriele Milani

Copyright (C) 2013 D. V. Bambill and C. A. Rossit. This is an open access article distributed under the Creative Commons Attribution License, which permits unrestricted use, distribution, and reproduction in any medium, provided the original work is properly cited.

\begin{abstract}
As it is known, the problems of free transverse vibrations and instability under in-plane loads of a plate are two different technological situations that have similarities in their approach to elastic solution. In fact, they are two eigenvalue problems in which we analyze the equilibrium situation of the plate in configurations which differ very slightly from the original, undeformed configuration. They are coupled in the event where in-plane forces are applied to the edges of the transversely vibrating plate. The presence of forces can have a significant effect on structural and mechanical performance and should be taken into account in the formulation of the dynamic problem. In this study, distributed forces of linear variation are considered and their influence on the natural frequencies and corresponding normal modes of transverse vibration is analyzed. It also analyzes their impact for the case of vibration control. The forces' magnitude is varied and the first natural frequencies of transverse vibration of rectangular thin plates with different combinations of edge conditions are obtained. The critical values of the forces which cause instability are also obtained. Due to the analytical complexity of the problem under study, the Ritz method is employed. Some numerical examples are presented.
\end{abstract}

\section{Introduction}

The transverse-free vibrations and buckling of plates which are subjected to edge loads acting in their middle planes are areas of research which have received a great deal of attention in the past century.

As it was stated experimentally by Hearmon [1] for the case of a beam, bifurcation buckling may be regarded as a special case of the vibration problem, that is, determining the in-plane stresses which cause vibration frequencies to reduce to zero.

Most of the work has dealt with rectangular plates having uniformly distributed in-plane edge loads. In that case, the governing differential equations of motion and equilibrium have constant coefficients, yielding exact solutions for frequencies and buckling loads straightforwardly when two opposite edges of the plates are simply supported.

Many researchers have analyzed both the buckling and vibration of rectangular plates subjected to in-plane stress field. Among them, one can mention Kang and Leissa [2]; Leissa and Kang [3]; Bassily and Dickinson [4]; Dickinson [5]; Kielb and Han [6]; Kaldas and Dickinson [7].

For the linearly varying loading, the governing differential equations have variable coefficients.

Leissa and Kang [3] found exact solutions for the free vibration and buckling problems of the SS-C-SS-C isotropic plate loaded at its simply supported edges by linearly varying in-plane stresses.

They also found the exact solution [8] for the buckling of rectangular plates having linearly varying in-plane loading on two opposite simply supported edges, with different boundary conditions at the other opposite edges.

Within the realm of the classical theory of plates, the case of buckling and vibrations problems for all the possibilities of boundary conditions and linearly varying in-plane forces offers considerable difficulty. This is the reason why it is quite common to make use of the Ritz variational method. 


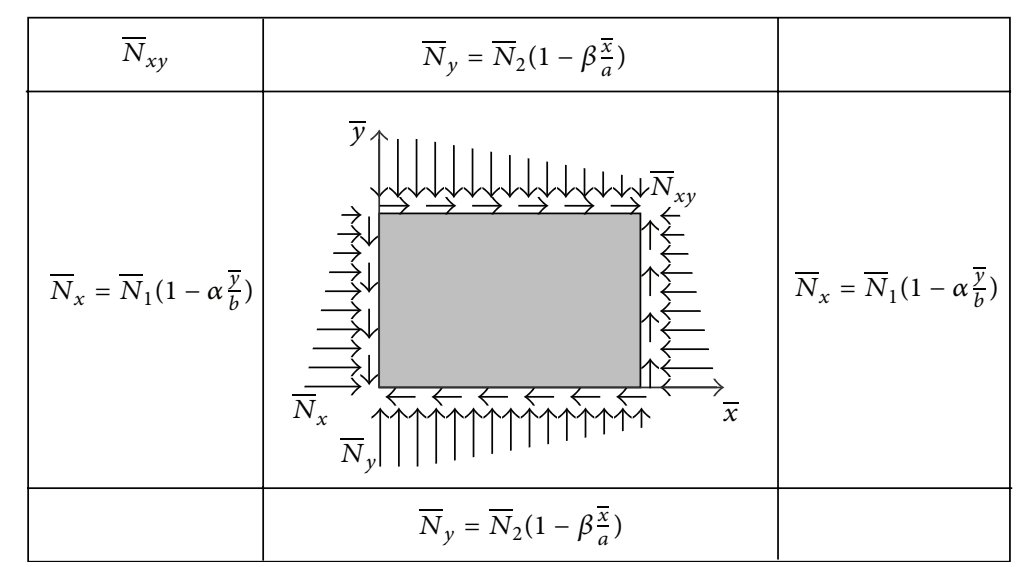

Figure 1: Rectangular vibrating plate subjected to in-plane loads: $\bar{N}_{x}, \bar{N}_{y}$, and $\bar{N}_{x y}$.

\section{Approximate Analytical Solution}

In the case of a transversely vibrating, thin, isotropic plate subjected to in-plane forces $N_{x}, N_{y}$, and $N_{x y}$, (Figure 1 and (5)), the maximum value of the potential energy due to bending deformation is

$$
\begin{aligned}
U_{\max }=\frac{1}{2} D \int_{A}[ & \left(\frac{\partial^{2} W}{\partial \bar{x}^{2}}+\frac{\partial^{2} W}{\partial \bar{y}^{2}}\right)^{2}+2(1-v) \\
& \left.\times\left(\frac{\partial^{2} W}{\partial \bar{x}^{2}} \frac{\partial^{2} W}{\partial \bar{y}^{2}}-\left(\frac{\partial^{2} W}{\partial \bar{x} \partial \bar{y}}\right)^{2}\right)\right] d \bar{x} d \bar{y}
\end{aligned}
$$

where $W=W(x, y)$ is the deflection amplitude of the middle plane of the plate, $D$ is the well known flexural rigidity $D=$ $E h^{3} / 12\left(1-v^{2}\right), E$ is the Young modulus, and $v$ is the Poisson coefficient.

While the maximum of the kinetic energy is

$$
T_{\max }=\frac{1}{2} \rho \omega^{2} \int_{A} h W^{2} d \bar{x} d \bar{y}
$$

where $\rho$ is the density of the plate material, $\omega$ is the circular frequency, and $h$ is the thickness of the plate.

And the maximum potential energy of the internal stresses caused by the in-plane loading is

$$
\begin{array}{r}
\tau_{N}=\frac{1}{2} \int_{A_{P}}\left(\bar{N}_{x}\left(\frac{\partial W}{\partial \bar{x}}\right)^{2}+\bar{N}_{y}\left(\frac{\partial W}{\partial \bar{y}}\right)^{2}\right. \\
\left.+2 \bar{N}_{x y} \frac{\partial W}{\partial \bar{x}} \frac{\partial W}{\partial \bar{y}}\right) d \bar{x} d \bar{y}
\end{array}
$$

The lengths of the sides of the rectangular plate are $a$ in the $x$ direction and $b$ in the $y$ direction. The coordinates are written in the dimensionless form as follows:

$$
x=\frac{\bar{x}}{a}, \quad y=\frac{\bar{y}}{b}
$$
[9]):

And the in-plane forces are expressed as (Bambill et al.

$$
N_{x}=\frac{\bar{N}_{x} b^{2}}{D}, \quad N_{y}=\frac{\bar{N}_{y} b^{2}}{D}, \quad N_{x y}=\frac{\bar{N}_{x y} b^{2}}{D} .
$$

Then, the governing functional of the system is

$$
\begin{aligned}
J[W]=\frac{1}{2} D\left[\frac{b}{a^{3}} \int_{A} N_{x}\left(\frac{\partial W}{\partial x}\right)^{2} d x d y\right. \\
+\frac{1}{a b} \int_{A} N_{y}\left(\frac{\partial W}{\partial y}\right)^{2} d x d y \\
\left.+\frac{2}{a^{2}} \int_{A} N_{x y} \frac{\partial W}{\partial x} \frac{\partial W}{\partial y} d x d y\right] \\
+\frac{1}{2} D\left[\frac{b}{a^{3}} \int_{A}\left(\frac{\partial^{2} W}{\partial x^{2}}\right)^{2} d x d y\right. \\
+\frac{2 v}{a b} \int_{A} \frac{\partial^{2} W}{\partial x^{2}} \frac{\partial^{2} W}{\partial y^{2}} d x d y \\
+\frac{a}{b^{3}} \int_{A}\left(\frac{\partial^{2} W}{\partial y^{2}}\right)^{2} d x d y \\
\left.+\frac{2(1-v)}{a b} \int_{A}\left(\frac{\partial^{2} W}{\partial x \partial y}\right)^{2} d x d y\right] \\
+\int_{A} h W^{2} d x d y .
\end{aligned}
$$

Equation (6) satisfies, if $W$ is the exact solution, the condition:

$$
\delta J[W]=0 .
$$


TABLE 1: The first six natural frequency coefficients $\Omega_{i}$ for a C-C-F-F plate under general in-plane loading.

\begin{tabular}{|c|c|c|c|c|c|}
\hline & \multicolumn{5}{|c|}{$N / N_{\text {crit }}$} \\
\hline & 0 & 0.25 & 0.5 & 0.75 & 1 \\
\hline \multicolumn{6}{|c|}{$\lambda=a / b=0.75 ; N_{\text {crit }}=5.95168$} \\
\hline$\Omega_{1}$ & 5.39789 & 4.75442 & 3.95041 & 2.84503 & 0 \\
\hline$\Omega_{2}$ & 15.7547 & 15.2037 & 14.5835 & 13.8967 & 13.1443 \\
\hline$\Omega_{3}$ & 23.8207 & 23.9071 & 24.0071 & 24.1145 & 24.2241 \\
\hline$\Omega_{4}$ & 36.3999 & 36.2826 & 36.1113 & 35.7654 & 35.1888 \\
\hline$\Omega_{5}$ & 38.1446 & 37.5193 & 36.9334 & 36.5072 & 36.2961 \\
\hline$\Omega_{6}$ & 59.7605 & 59.5098 & 59.2367 & 58.9439 & 58.6327 \\
\hline \multicolumn{6}{|c|}{$\lambda=a / b=1 ; N_{\text {crit }}=3.69137$} \\
\hline$\Omega_{1}$ & 6.93254 & 6.06065 & 4.99441 & 3.56413 & 0 \\
\hline$\Omega_{2}$ & 23.9780 & 23.9778 & 23.6766 & 23.0314 & 22.1751 \\
\hline$\Omega_{3}$ & 26.6265 & 26.0033 & 25.6318 & 25.5511 & 25.6238 \\
\hline$\Omega_{4}$ & 47.7179 & 47.4465 & 47.1607 & 46.8601 & 46.5440 \\
\hline$\Omega_{5}$ & 62.8830 & 62.6774 & 62.3443 & 61.9069 & 61.3970 \\
\hline$\Omega_{6}$ & 65.6818 & 65.4396 & 65.3185 & 65.2954 & 65.3384 \\
\hline \multicolumn{6}{|c|}{$\lambda=a / b=2 ; N_{\text {crit }}=1.85222$} \\
\hline$\Omega_{1}$ & 17.1515 & 14.9296 & 12.2458 & 8.69504 & 0 \\
\hline$\Omega_{2}$ & 36.4410 & 36.0647 & 35.6415 & 35.1668 & 34.6357 \\
\hline$\Omega_{3}$ & 73.5943 & 73.8555 & 74.0906 & 74.2962 & 74.4680 \\
\hline$\Omega_{4}$ & 91.1002 & 89.5702 & 88.0081 & 86.4144 & 84.7901 \\
\hline$\Omega_{5}$ & 115.363 & 114.262 & 113.147 & 112.021 & 110.886 \\
\hline$\Omega_{6}$ & 131.962 & 132.267 & 132.566 & 132.855 & 133.130 \\
\hline
\end{tabular}

TABLE 2: The first six natural frequency coefficients $\Omega_{i}$ for a C-C-SSSS plate under uniform $N_{x}$ loading $(\alpha=0)$.

\begin{tabular}{lcccccc}
\hline$\lambda=a / b=1$ & \multicolumn{6}{c}{$N / N_{\text {crit }}$} \\
$N_{\text {crit }}=61.4151$ & 0 & 0.1 & 0.25 & 0.5 & 0.75 & 1 \\
\hline$\Omega_{1}$ & 27.0542 & 25.7489 & 23.6368 & 19.5326 & 14.0587 & 0 \\
$\Omega_{2}$ & 60.5387 & 58.4465 & 54.9733 & 48.6153 & 41.2865 & 32.4139 \\
$\Omega_{3}$ & 60.7863 & 60.1238 & 59.2827 & 57.8385 & 56.3769 & 54.8441 \\
$\Omega_{4}$ & 92.8371 & 91.4269 & 89.2672 & 85.5386 & 81.6286 & 77.5081 \\
$\Omega_{5}$ & 114.557 & 112.082 & 108.152 & 101.257 & 93.8564 & 85.8259 \\
$\Omega_{6}$ & 114.704 & 114.350 & 113.923 & 113.207 & 112.485 & 111.755 \\
\hline
\end{tabular}

Following the Ritz method, the expression of the deflection of the plate is approximated in the form of a truncated series:

$$
W \cong W_{a}(x, y)=\sum_{m=1}^{M} \sum_{n=1}^{N} A_{m n} X_{m}(x) Y_{n}(y),
$$

where $X_{m}(x)$ and $Y_{n}(y)$ are the characteristic functions for the normal modes of vibration of beams with end conditions nominally similar to those of the opposite edges of the plate in each coordinate direction [10]. Consequently, they satisfy the essential boundary conditions, as the method requires.
The variational equation (7) is replaced by the homogeneous linear system of equations:

$$
\frac{\partial J\left[W_{a}\right]}{\partial A_{q l}}=0, \quad q=\{1,2, \ldots M\} ; l=\{1,2, \ldots N\},
$$

and using nondimensional variables, becomes

$$
\frac{a b}{D} \frac{\partial J\left[W_{a}\right]}{\partial A_{q l}}=0
$$

Finally, one obtains a homogeneous linear system of equations in terms of the $A_{q l}$ 's parameters.

The nontriviality condition of the system (10) requires the determinant to be zero:

$$
\left|S \cdot\left[\tau_{q l m n}\right]+\left[u_{q l m n}\right]-\Omega^{2} \cdot\left[t_{q l m n}\right]\right|=0,
$$

where $\Omega_{m n}=\omega_{m n} a^{2} \sqrt{\rho h / D}$ are the frequency coefficients.

The elements of the matrices involved in (11) are given by

$$
\begin{aligned}
\tau_{q l m n}= & \frac{1}{\lambda^{2}} \int_{A_{n}} N_{x}^{*}\left(\frac{d X_{q}}{d x} \frac{d X_{m}}{d x}\right)\left(Y_{l} Y_{n}\right) d x d y \\
& +\int_{A_{n}} N_{y}^{*}\left(X_{q} X_{m}\right)\left(\frac{d Y_{l}}{d y} \frac{d Y_{n}}{d y}\right) d x d y \\
& \times \frac{1}{\lambda} \int_{A_{n}} N_{x y}^{*}\left(X_{q} \frac{d X_{m}}{d x} \frac{d Y_{l}}{d y} Y_{n}\right. \\
& \left.+\frac{d X_{q}}{d x} X_{m} Y_{l} \frac{d Y_{n}}{d y}\right) d x d y,
\end{aligned}
$$

where $\lambda=a / b$ is the aspect ratio

$$
N_{x}=S \cdot N_{x}^{*}, \quad N_{y}=S \cdot N_{y}^{*}, \quad N_{x y}=S \cdot N_{x y}^{*},
$$

where $S$ is a factor that indicates the magnitude of the in-plane loading system, regarding the relative value of the forces. Consider

$$
\begin{aligned}
u_{q l m n}= & \lambda^{-2} \int_{A}\left(\frac{d^{2} X_{q}}{d x^{2}} \frac{d^{2} X_{m}}{d x^{2}}\right)\left(Y_{l} Y_{n}\right) d x d y \\
+ & v \int_{A}\left[\left(X_{q} \frac{d^{2} X_{m}}{d x^{2}}\right)\left(\frac{d^{2} Y_{l}}{d y^{2}} Y_{n}\right)\right. \\
& \left.+\left(\frac{d^{2} X_{q}}{d x^{2}} X_{m}\right)\left(Y_{l} \frac{d^{2} Y_{n}}{d y^{2}}\right)\right] d x d y \\
+ & \lambda^{2} \int_{A}\left(X_{q} X_{m}\right)\left(\frac{d^{2} Y_{l}}{d y^{2}} \frac{d^{2} Y_{n}}{d y^{2}}\right) d x d y \\
+ & 2(1-v) \int_{A}\left(\frac{d X_{q}}{d x} \frac{d X_{m}}{d x}\right)\left(\frac{d Y_{l}}{d y} \frac{d Y_{n}}{d y}\right) d x d y, \\
& t_{q l m n}=\lambda^{-2} \int_{A}\left(X_{q} X_{m}\right)\left(Y_{l} Y_{n}\right) d x d y .
\end{aligned}
$$

As it is known, the condition $\Omega=0$ in (11) yields the critical value of the in-plane loading. 
TABLE 3: The first six natural frequency coefficients $\Omega_{i}$ for a C-C-SS-SS plate under uniform shear $N_{x y}$ loading.

\begin{tabular}{lcccccrrr}
\hline$\lambda=a / b=1.5$ & \multicolumn{9}{c}{$N / N_{\text {crit }}$} \\
$N_{\text {crit }}=89.8137$ & 0 & 0.1 & 0.25 & 0.4 & 0.5 & 0.75 & 0.9 \\
\hline$\Omega_{1}$ & 44.8904 & 44.7005 & 43.7991 & 42.4070 & 40.2822 & 32.4323 & 22.3996 \\
$\Omega_{2}$ & 76.5451 & 76.1864 & 74.3165 & 70.7817 & 67.4142 & 54.4376 & 41.3590 & 27.7956 \\
$\Omega_{3}$ & 122.319 & 122.349 & 122.548 & 121.465 & 117.487 & 104.882 & 95.5807 & 88.7020 \\
$\Omega_{4}$ & 129.393 & 128.871 & 126.129 & 122.871 & 123.101 & 123.415 & 123.108 & 122.473 \\
$\Omega_{5}$ & 152.529 & 152.707 & 153.680 & 155.033 & 155.912 & 157.429 & 157.633 & 157.273 \\
$\Omega_{6}$ & 202.615 & 200.101 & 194.748 & 188.611 & 184.103 & 171.463 & 163.073 & 214.018 \\
\hline
\end{tabular}

TABLE 4: Comparison of nondimensional critical buckling loads $N_{\text {crit }}=\bar{N}_{\text {crit }} b^{2} / D$ for a SS-C-SS-C plate.

\begin{tabular}{|c|c|c|c|c|c|c|c|c|}
\hline \multirow{2}{*}{ Load $(\alpha)$} & \multirow{2}{*}{ Solution } & \multicolumn{7}{|c|}{$\lambda=a / b$} \\
\hline & & 0.4 & 0.5 & 0.6 & 0.7 & 0.8 & 0.9 & 1.0 \\
\hline \multirow{5}{*}{0} & [A] $M=5$ & 93.3059 & 75.9452 & 69.6553 & 69.1116 & 72.0966 & 77.5543 & 75.9452 \\
\hline & [A] $M=10$ & 93.2555 & 75.9146 & 69.6351 & 69.0972 & 72.0859 & 77.5460 & 75.9146 \\
\hline & [A] $M=15$ & 93.2477 & 75.9105 & 69.6323 & 69.0954 & 72.0846 & 77.5450 & 75.9105 \\
\hline & [A] $M=20$ & 93.2476 & 75.9101 & 69.6323 & 69.09531 & 72.0844 & 77.5449 & 75.9101 \\
\hline & [B] & 93.247 & 75.910 & 69.632 & 69.095 & 72.084 & 77.545 & 75.910 \\
\hline \multirow{5}{*}{1} & [A] $M=5$ & 174.533 & 145.286 & 134.809 & 134.624 & 140.981 & 152.024 & 145.286 \\
\hline & [A] $M=10$ & 174.395 & 145.215 & 134.765 & 134.593 & 140.958 & 152.007 & 145.215 \\
\hline & [A] $M=15$ & 174.379 & 145.207 & 134.760 & 134.590 & 140.956 & 152.005 & 145.207 \\
\hline & [A] $M=20$ & 174.377 & 145.206 & 134.760 & 134.590 & 140.956 & 152.005 & 145.206 \\
\hline & [B] & 174.4 & 145.2 & 134.8 & 134.6 & 141.0 & 152.0 & 145.2 \\
\hline \multirow{5}{*}{2} & [A] $M=5$ & 401.518 & 392.147 & 412.162 & 424.140 & 401.518 & 392.143 & 392.147 \\
\hline & [A] $M=10$ & 400.478 & 391.589 & 411.812 & 422.594 & 400.478 & 391.398 & 391.589 \\
\hline & [A] $M=15$ & 400.410 & 391.548 & 411.790 & 422.490 & 400.410 & 391.351 & 391.548 \\
\hline & [A] $M=20$ & 400.399 & 391.548 & 411.787 & 422.472 & 400.399 & 391.343 & 391.547 \\
\hline & {$[\mathrm{B}]$} & 400.4 & 391.5 & 411.8 & 422.5 & 400.4 & - & 391.5 \\
\hline
\end{tabular}

[A]: present approach with different $M=N$ and [B]: [3].

\section{Numerical Evaluations}

Hearmon [1] has experimented on a fixed-free strip. Admittedly, the problem is analytically simpler in the case of one-dimensional domains. As an example, let us try with a pinned-pinned transversely vibrating beam, subjected to an axial compressive force $P$. The expression of the frequency coefficient is

$$
\begin{aligned}
\Omega_{n} & =\sqrt{\frac{\rho A_{0}}{\mathrm{EI}}} L^{2} \omega_{n} \\
& =(n \pi)^{2} \sqrt{\left[1-\frac{P L^{2}}{\mathrm{EI}(n \pi)^{2}}\right]} ; \quad \text { with } n=1,2,3,4 \ldots,
\end{aligned}
$$

where $\rho$ is the density of the material, $A_{0}$ is the cross-section, $L$ is the length, and EI the flexural rigidity of the beam.

All the Euler buckling loads are determined making zero expression (15). For $n=1$, the critical buckling load of the beam, $P_{\text {crit }}$, is obtained.

Plotting the values $\Omega_{n}=(n \pi)^{2} \sqrt{\left(1-\left(P / n^{2} P_{\text {crit }}\right)\right)}$ of the first three frequency coefficients depending upon the ratio $P / P_{\text {crit }}$ yield regular curves as is shown in Figure 2 . The

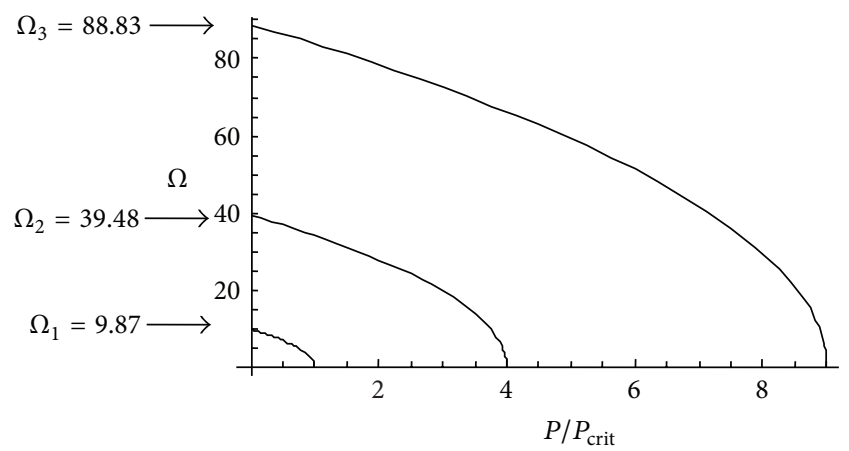

FIgURE 2: Curves of the frequency coefficients of transverse vibration for a pinned-pinned beam under axial compressive load $P$.

presence of the compressive axial load $P$ does not alter the order of the modal shapes of the beam.

In the case of a plate, in general, and due to the bidimensional behavior induced by the torsional rigidity, the compressive in-plane load may alter both the order and shape of the modal shapes associated to each natural frequency. 
TABLE 5: Comparison of the first six natural frequency coefficients for a SS-C-SS-C plate under bending moment in $x$-direction $(\alpha=2)$.

\begin{tabular}{|c|c|c|c|c|c|c|c|c|}
\hline$\lambda=a / b$ & $N / N_{\text {crit }}$ & Solution & $\Omega_{1}$ & $\Omega_{2}$ & $\Omega_{3}$ & $\Omega_{4}$ & $\Omega_{5}$ & $\Omega_{6}$ \\
\hline \multirow{6}{*}{0.5} & \multirow{2}{*}{0} & [A] & 13.6858 & 23.6465 & 38.6942 & 42.5871 & 51.6767 & 58.647 \\
\hline & & [B] & 13.69 & 23.65 & - & 42.59 & 51.67 & - \\
\hline & \multirow{2}{*}{0.5} & {$[\mathrm{~A}]$} & 11.4936 & 24.152 & 37.9343 & 38.8757 & 52.632 & 58.7048 \\
\hline & & [B] & 11.49 & 24.15 & 37.93 & - & 52.63 & - \\
\hline & \multirow{2}{*}{0.95} & {$[\mathrm{~A}]$} & 3.92178 & 24.906 & 27.6496 & 39.3337 & 52.6657 & 58.8581 \\
\hline & & [B] & 3.926 & 24.91 & 27.65 & - & 52.66 & - \\
\hline \multirow{6}{*}{1} & \multirow{2}{*}{0} & {$[\mathrm{~A}]$} & 28.9509 & 54.7433 & 69.3271 & 94.5862 & 102.217 & 129.096 \\
\hline & & [B] & 28.95 & 54.74 & 69.33 & 94.59 & 102.2 & - \\
\hline & \multirow{2}{*}{0.5} & {$[\mathrm{~A}]$} & 27.4647 & 45.9744 & 69.6407 & 87.2215 & 96.6079 & 129.164 \\
\hline & & [B] & 27.47 & 45.97 & 69.65 & 87.22 & 96.61 & - \\
\hline & \multirow{2}{*}{0.95} & {$[\mathrm{~A}]$} & 15.6871 & 23.3557 & 46.9793 & 70.3963 & 99.624 & 110.599 \\
\hline & & [B] & 15.70 & 23.37 & 46.96 & 70.41 & 99.63 & 110.6 \\
\hline \multirow{6}{*}{2} & \multirow{2}{*}{0} & {$[\mathrm{~A}]$} & 95.2625 & 115.803 & 156.357 & 218.973 & 254.138 & 277.308 \\
\hline & & [B] & 95.26 & 115.8 & 156.4 & - & 254.1 & 277.3 \\
\hline & \multirow{2}{*}{0.5} & {$[\mathrm{~A}]$} & 94.7438 & 109.859 & 137.29 & 183.897 & 254.214 & 254.254 \\
\hline & & [B] & 94.76 & 109.9 & 137.3 & 183.9 & 254.2 & - \\
\hline & \multirow{2}{*}{0.95} & {$[\mathrm{~A}]$} & 62.7485 & 77.444 & 93.4166 & 93.4229 & 101.133 & 187.917 \\
\hline & & {$[\mathrm{B}]$} & 62.82 & 77.50 & 93.46 & 93.47 & - & - \\
\hline
\end{tabular}

[A]: present approach and [B]: [3].

\begin{tabular}{|l|l|l|l|}
\hline \multicolumn{1}{|c|}{$N / N_{\text {crit }}=0$} & \multicolumn{2}{c|}{$N / N_{\text {crit }}=0.1$} \\
\hline$\Omega_{1}=27.0542$ & $\Omega_{2}=60.5387$ & $\Omega_{1}=25.7489$ & $\Omega_{2}=58.4465$ \\
\hline$\Omega_{3}=60.7863$ & & & \\
\hline & & & \\
\hline & & & \\
\hline
\end{tabular}

FIgURE 3: Normal modes of vibration of a square C-C-SS-SS plate under uniform $N_{x}$ loading $\left(\lambda=a / b=1, N_{\text {crit }}=61.4151\right)$.

This situation has an important technological signification from the point of view of vibration control.

Certainly, the modal shape of a natural resonant frequency must be known in order to suppress it. In the case of in-plane loading, this shape can be different from the expected one.

Due to the quantity and variability of the parameters involved in the description of the behaviour of these kinds of structures, just a few representative cases will be considered to demonstrate the convenience of the procedure and the importance of the situation.

All the values are determined taking $M=N=15$ in (8).
Table 1 shows the values of the first natural frequency coefficients for a CCFF plate subjected to a general in-plane loading: linear load in $x$ direction $(\alpha=2)$-bending moment, constant load in $y$ direction $(\beta=0)$, and constant shear force $N_{x y}=N_{1}=N_{2}=N$ ).

In order to show the influence of the in-plane loading, the next two examples are presented.

Table 2 shows the natural frequency coefficients for a CC-SS-SS square plate under uniform compression in the $x$ direction $\left(N_{x}=N, \alpha=0\right.$, and $\left.N_{y}=N_{x y}=0\right)$.

Figure 3 shows that a minimal presence of in-plane loading ( $10 \%$ of the critical value) dramatically modifies the 


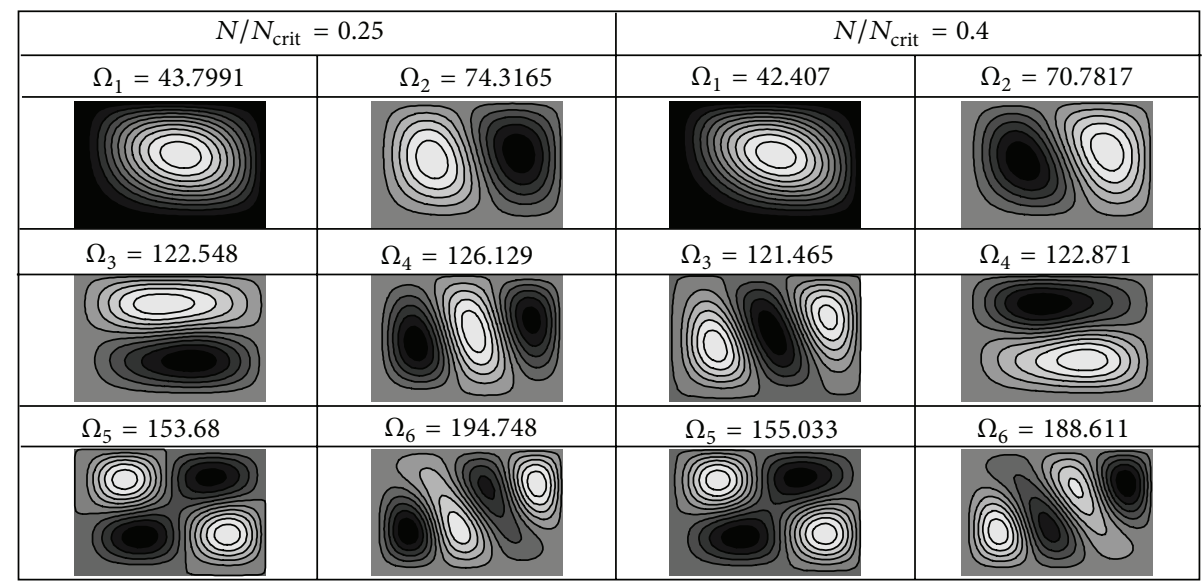

Figure 4: Normal modes of vibration of a rectangular C-C-SS-SS plate under uniform $N_{x y}$ loading $\left(\lambda=a / b=1.5, N_{\text {crit }}=89.8137\right)$.

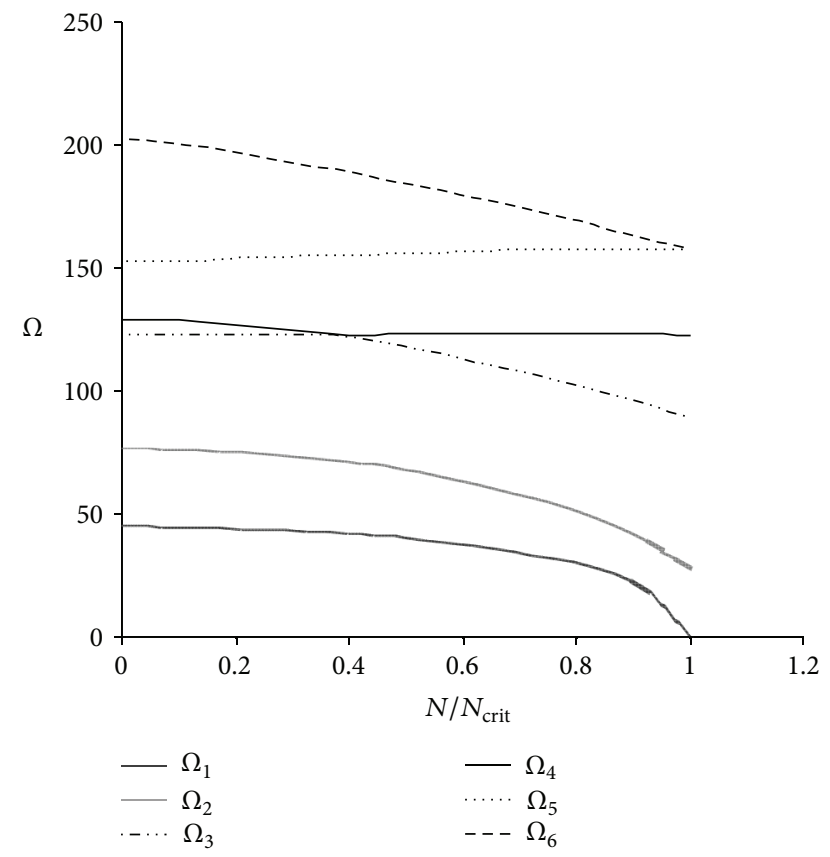

Figure 5: The first six natural frequency coefficients $\Omega_{i}$ for a C-CSS-SS plate under uniform shear $N_{x y}$ loading $\left(\lambda=a / b=1.5, N_{\text {crit }}=\right.$ 89.8137).

mode shapes, while changes in the values of frequencies may not be noticed $(0.3 \%$ in the sixth frequency). It is important to point out that the small load can be originated by thermal variations and restrictions on plane displacements imposed by the external supports.

Finally, Table 3 shows the results for a rectangular C-CSS-SS plate subjected to shear in-plane forces.

Figure 4 shows that the third and fourth natural frequencies interchange their normal modes as $N_{x y}$ increases. This situation is noticeable from Figure 5.

This means that for a given value of $N_{x y}$, between 0.25 and 0.4 of the critical value, there are two normal modes for the same natural frequency (repeated frequency). This is an important point in vibration control, since when repeated frequencies arise in a system, the related vibration mode shape cannot be uniquely determined. Any linear combination of the modes is still valid for the repeated frequency.

In order to evaluate the accuracy of the expounded procedure, comparison is made with the results obtained in [3] for a SS-C-SS-C plate loaded at its simply supported edges by linearly varying in-plane stresses (Tables 4 and 5).

In Table 4, values of $N_{\text {crit }}$ are compared for three different cases of the $x$ direction load: constant $(\alpha=0)$, linear with null value at one extreme $(\alpha=1)$, and bending moment $(\alpha=2)$, and different aspect radii of the plate. A convergence study is also made. As it can be seen, taking $M=N=15$ provides an excellent accuracy from an engineering viewpoint.

\section{Conclusions}

The classical, variational method of Ritz has been successfully used in the present study to obtain an approximate, yet quite accurate, solution to a difficult elastodynamics problem.

Natural frequencies and mode shapes of transverse vibration are obtained for a meaningful combination of the boundary conditions of a thin plate subjected to general inplane loads. The critical values of the in-plane forces which cause instability of the plates are also obtained.

The obtained values are the outcome of an algorithm, relatively simple to implement, [11] which allows studying these with only the assistance of a PC.

Additional complexities like orthotropic material characteristics can be taken into account [12].

The agreement with results available in the literature is excellent. Nevertheless, it is also possible to increase the number of terms in the summation on (8) to increase the accuracy.

No claim of originality is made, but it is hoped that the present work draws the attention to the effect that the presence of plane stress state may have on the effectiveness of vibration control on plates.

\section{Acknowledgments}

The present work has been sponsored by the Secretaría General de Ciencia y Tecnología of Universidad Nacional del Sur, UNS, at the Department of Engineering and by 
Consejo Nacional de Investigaciones Científicas y Técnicas, CONICET. The authors wish to thank Dr. D. H. Felix from Universidad Nacional del Sur.

\section{References}

[1] R. F. S. Hearmon, "The frequency of vibration and the elastic stability of a fixed-free strip," British Journal of Applied Physics, vol. 7, no. 11, pp. 405-407, 1956.

[2] J.-H. Kang and A. W. Leissa, "Vibration and buckling of SS-F-SS-F rectangular plates loaded by in-plane moments," International Journal of Stability and Dynamics, vol. 1, no. 4, pp. 527-543, 2001.

[3] A. W. Leissa and J.-H. Kang, "Exact solutions for vibration and buckling of an SS-C-SS-C rectangular plate loaded by linearly varying in-plane stresses," International Journal of Mechanical Sciences, vol. 44, no. 9, pp. 1925-1945, 2002.

[4] S. F. Bassily and S. M. Dickinson, "Buckling and lateral vibration of rectangular plates subject to in-plane loads-a Ritz approach," Journal of Sound and Vibration, vol. 24, no. 2, pp. 219-239, 1972.

[5] S. M. Dickinson, "The buckling and frequency of flexural vibration of rectangular isotropic and orthotropic plates using Rayleigh's method," Journal of Sound and Vibration, vol. 61, no. 1, pp. 1-8, 1978.

[6] R. E. Kielb and L. S. Han, "Vibration and buckling of rectangular plates under in-plane hydrostatic loading," Journal of Sound and Vibration, vol. 70, no. 4, pp. 543-555, 1980.

[7] M. M. Kaldas and S. M. Dickinson, "Vibration and buckling calculations for rectangular plates subject to complicated inplane stress distributions by using numerical integration in a Rayleigh-Ritz analysis," Journal of Sound and Vibration, vol. 75, no. 2, pp. 151-162, 1981.

[8] J.-H. Kang and A. W. Leissa, "Exact solutions for the buckling of rectangular plates having linearly varying in-plane loading on two opposite simply supported edges," International Journal of Solids and Structures, vol. 42, no. 14, pp. 4220-4238, 2005.

[9] D. V. Bambill, C. A. Rossit, and D. H. Felix, "Comments on 'Buckling behavior of a graphite/epoxy composite plate under parabolic variation of axial loads,' International Journal of Mechanical Sciences, vol. 47, no. 9, pp. 1473-1474, 2005.

[10] R. P. Felgar Jr., Formulas for Integrals Containing Characteristic Functions of a Vibrating Beam, Circular, no. 14, The University of Texas Publication, 1951.

[11] D. H. Felix, D. V. Bambill, and C. A. Rossit, "Desarrollo de un algoritmo de cálculo para la implementación del método de Rayleigh-Ritz en el cálculo de frecuencias naturales de vibración de placas rectangulares con complejidades diversas," Revista Internacional de Métodos Numéricos para Cálculo y Diseño en Ingeniería, vol. 20, no. 2, pp. 123-138, 2004.

[12] D. H. Felix, D. V. Bambill, and C. A. Rossit, "A note on buckling and vibration of clamped orthotopic plates under in-plane loads," Structural Engineering and Mechanics, vol. 39, no. 1, pp. 115-123, 2011. 

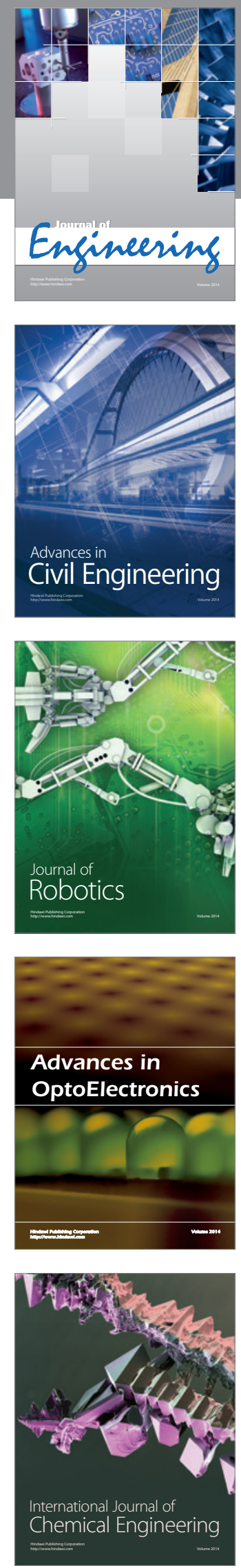

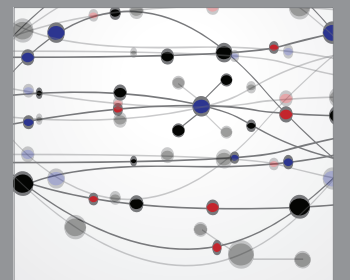

The Scientific World Journal
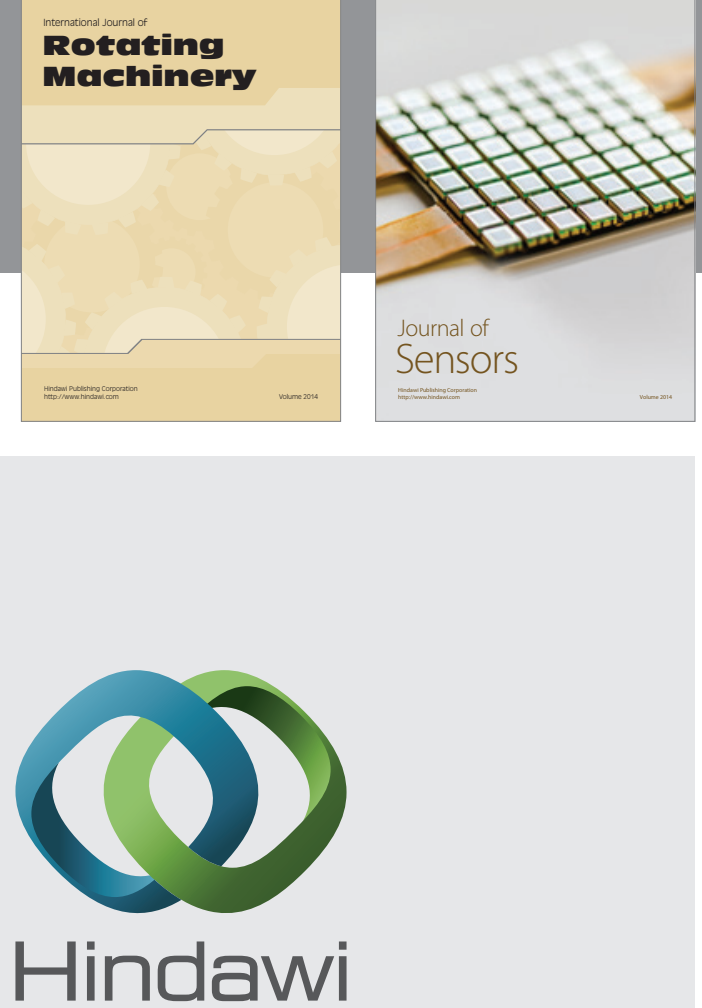

Submit your manuscripts at http://www.hindawi.com
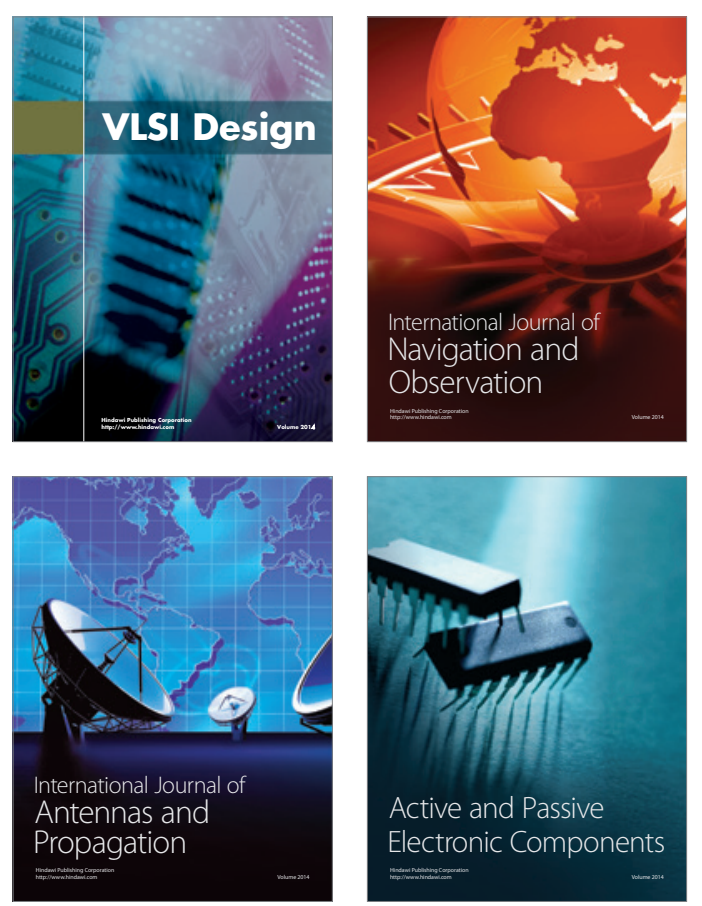
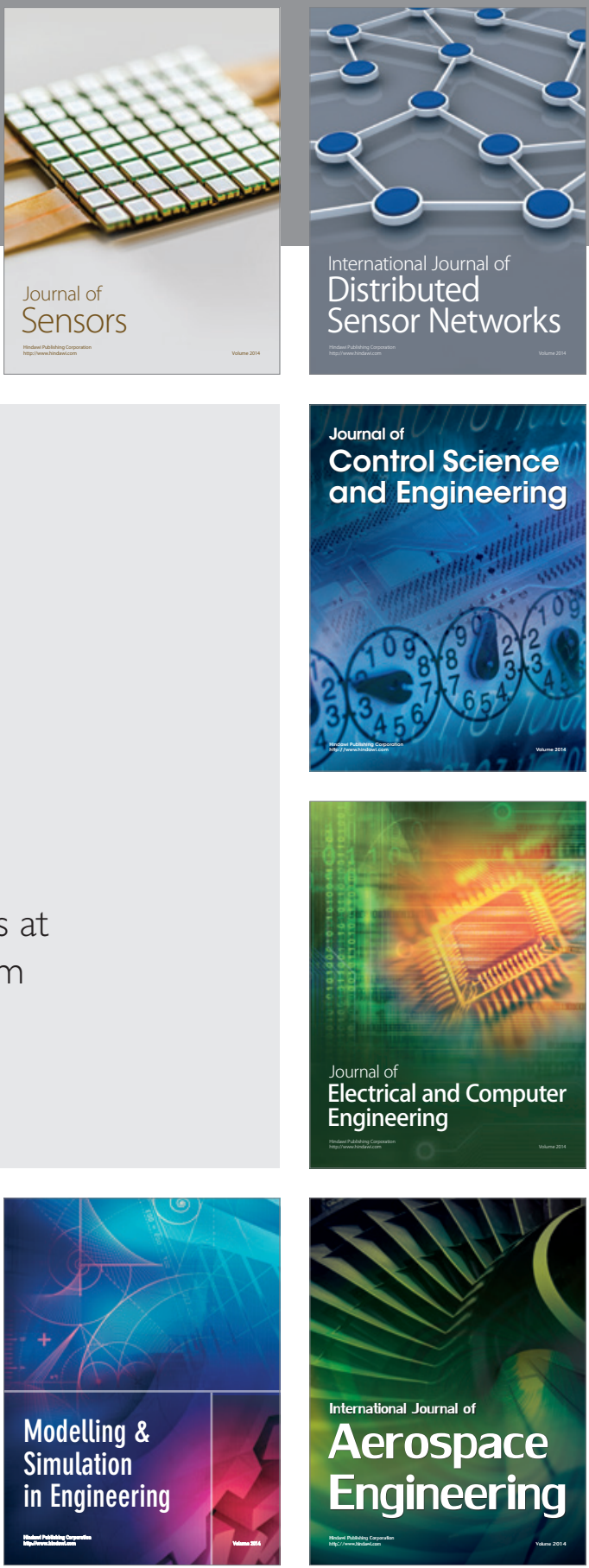

Journal of

Control Science

and Engineering
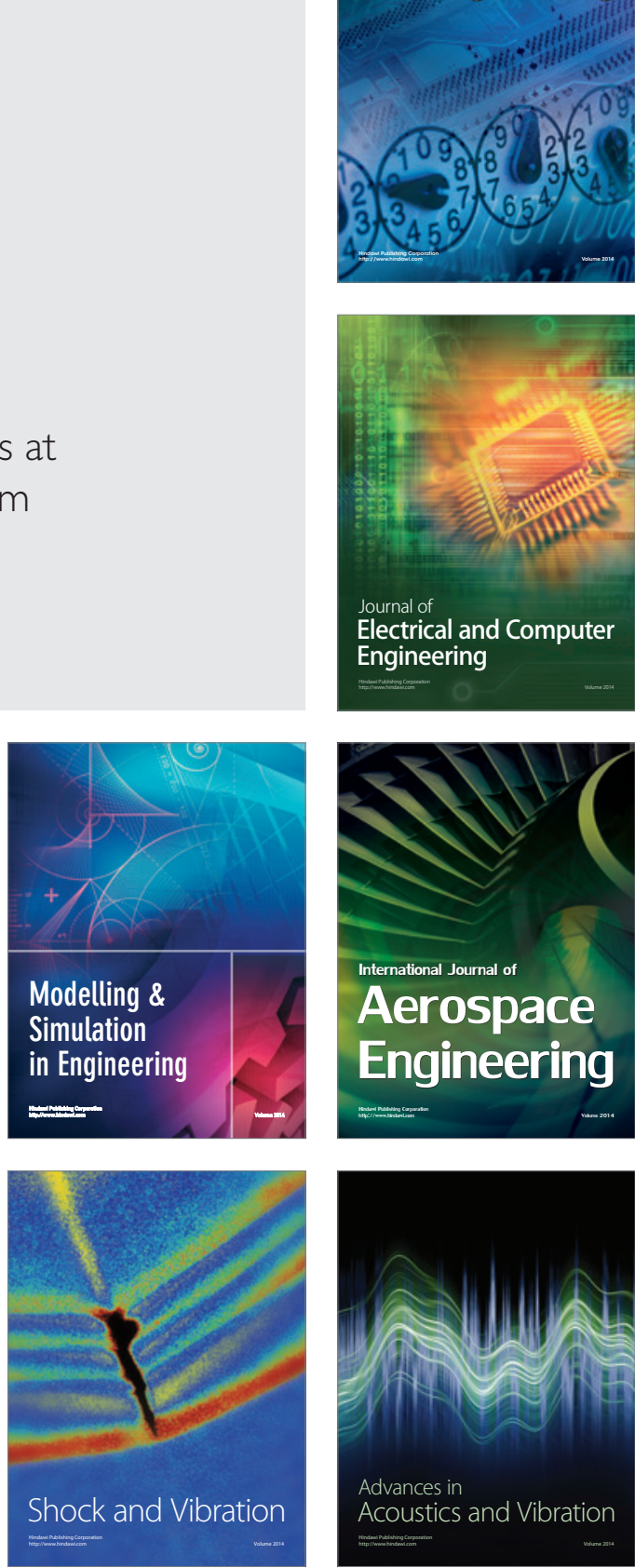